\title{
Crianças usuárias de lente de contato nos serviços público e privado: análise comparativa
}

\author{
Pediatric contact lens users in public and private services: comparative analysis
}

\author{
Daniela Araújo Toscano ${ }^{1}$ \\ Ana Cláudia Tabosa Florêncio ${ }^{2}$ \\ Maria da Conceição Sales ${ }^{3}$ \\ Márcia Trovão Duarte Cavalcanti ${ }^{4}$ \\ Daniela Almeida Lyra Antunes ${ }^{5}$
}

\begin{tabular}{|l|}
\hline RESUMO \\
\hline Objetivos: Analisar as indicações, tipo, complicações do uso de lentes \\
de contato e acuidade visual em crianças de serviços de Oftalmologia \\
público e privado. Métodos: Os dados dos prontuários de 59 crianças \\
usuárias de lentes de contato em serviço privado (Hospital de Olhos de \\
Pernambuco - Grupo 1), e 43 no serviço público (Fundação Altino \\
Ventura - Grupo 2), foram analisados. A coleta de dados incluiu carac- \\
terísticas sociodemográficas, idade da primeira consulta, indicação do \\
uso da lente, tipo de lente, complicações e acuidade visual. Resultados: \\
As mais comuns indicações do uso de lente de contato no grupo 1 foram: \\
ametropia (55,9\%), anisometropia (18,6\%) e esotropia (16,9\%). Neste \\
grupo o leucoma e phthisis não estavam presentes. No grupo 2, as \\
indicações mais comuns foram: anisometropia (23,2\%), ametropia e \\
leucoma (18,6\%) cada, e phthisis (16,3\%). A esotropia não apareceu no \\
grupo 2. O tipo de lente de contato mais prescrita foi a gelatinosa de uso \\
permanente (não descartável) no grupo 1 (45,8\%) e no grupo 2 (32,6\%). \\
A complicação mais encontrada no grupo 1 foi desconforto (33,3\%) e no \\
grupo 2 perda da lente (60\%). Conclusões: A indicação de ametropia \\
predominou nos pacientes privados e as anisometropias nos públicos. \\
O tipo de lente de contato mais prescrita nos dois grupos foi a gelatinosa \\
de uso permanente. A complicação mais frequente no grupo 1 foi des- \\
conforto e no grupo 2 perda da lente. A acuidade visual na maioria dos \\
pacientes manteve-se.
\end{tabular}

Descritores: Lente de contato/efeitos adversos; Acuidade visual; Crianças; Hospitais públicos; Hospitais privados; Análise comparativa
Trabalho realizado na Fundação Altino Ventura - FAV - Recife (PE) - Brasil.

${ }^{1}$ Aluna do Curso de Especialização em Oftalmologia da Fundação Altino Ventura - FAV - Recife (PE) - Brasil. ${ }^{2}$ Preceptora do Departamento de Lente de Contato da FAV - Recife (PE) - Brasil.

${ }^{3}$ Coordenadora do Departamento de Lente de Contato da FAV - Recife (PE) - Brasil.

${ }^{4}$ Aluna do Curso de Especialização em Oftalmologia da FAV - Recife (PE) - Brasil.

${ }_{5}^{5}$ Aluna do Curso de Especialização em Oftalmologia da FAV - Recife (PE) - Brasil.

Endereço para correspondência: Rua da Soledade, 170 - Recife (PE) CEP 50070-040

E-mail: danielatoscano@globo.com

Recebido para publicação em 02.11.2005

Última versão recebida em 22.01.2007

Aprovação em 16.02.2009

\section{INTRODUÇÃO}

O sucesso ou falha na adaptação de lente de contato em crianças continua, ainda, dependendo do entendimento, cooperação e persistência dos pais e da paciência e dedicação do profissional e auxiliares ${ }^{(1)}$.

O problema visual mais comum em crianças é o erro refrativo, e os óculos são o primeiro meio para corrigi-los ${ }^{(2)}$. A grande diferença entre o uso de lentes de contato em crianças, jovens e adultos é quanto à área de tratamento da ambliopia, que é importante para a criança ${ }^{(2)}$. Evitando a ambliopia a intenção é desenvolver a binocularidade, condição indispensável para a manutenção da acuidade visual ${ }^{(3)}$.

As lentes de contato rígidas gás permeáveis com altos DKs são as preferidas, mas entre 3 e 5 anos de idade, geralmente as crianças não aceitam lentes de contato rígidas, são adaptados então, lentes hidrofílicas de uso prolongado $^{(1)}$. 
As lentes de contato em crianças são indicadas geralmente quando com os óculos não se consegue visão binocular ou não se obtém melhora da acuidade visual ${ }^{(1)}$. As principais indicações são afacia, anisometropia, miopia, hipermetropia, astigmatismo irregular e nistagmo. Lentes de contato cosméticas são usadas para aniridria, albinismo, no tratamento de ambliopia e em opacidades corneanas ${ }^{(4-5)}$.

Devido a carência de trabalhos sobre este assunto, houve um interesse de analisar-se as diferenças existentes quanto à acuidade visual com óculos, as indicações, tipos de lentes de contato e as complicações das crianças usuárias de lentes de contatos em um serviço público e privado.

\section{MÉTODOS}

Foram analisados, de forma retrospectiva, prontuários de crianças usuárias de lente de contato em um serviço privado (Hospital de Olhos de Pernambuco - grupo 1) e serviço público (Fundação Altino Ventura - grupo 2), no período de 1997 a 2002. Utilizou-se uma amostra aleatória representativa no grupo 1, devido ao grande número de pacientes. No grupo 2 , utilizou-se todos os pacientes.

Analisou-se os dados referentes ao sexo, idade, idade da primeira consulta, acuidade visual, indicação da lente de contato, tipo de lente utilizada e complicações.

Os resultados qualitativos foram expressos em suas frequências, e os quantitativos por suas médias e desvios padrão. A comparação entre as indicações, tipo de lente e complicações referente aos dois grupos, foi realizada por teste exato de Fisher-Freeman-Halton. Aceitou-se o nível de significância de $\mathrm{p}<0,05$.

\section{RESULTADOS}

No grupo 1 foram analisados os dados de 59 prontuários e no grupo 2,43 .

Dos 3.127 prontuários analisados no grupo 1, 59 (1,9\%) eram crianças (menores que 12 anos completos). No grupo 2, dos 1.627 prontuários analisados foram encontrados $43(2,6 \%)$ crianças.

A média da idade da primeira consulta no grupo 1 foi de 9,8 anos $\pm 2,45$, variando de 3 a 12 anos. No grupo 2 a média foi de $9,8 \pm 2,3$, variando de 4 a 12 anos. A média da idade atual das crianças é de 11,4 $\pm 2,7$ anos no grupo 1, e 11,7 $\pm 2,0$ anos no grupo 2.

Vinte e cinco crianças $(42,4 \%)$ eram do sexo masculino e $34(57,6 \%)$ do feminino no grupo 1 . No grupo 2 a distribuição do sexo foi $24(55,8 \%)$ masculino e $19(44,2 \%)$ feminino.

A distribuição das frequências das indicações do uso da lente de contato nos grupos separadamente está na tabela 1 .

As frequências dos tipos de lente de contato estão na tabela 2.

A distribuição das frequências das complicações está na tabela 3 .

\begin{tabular}{|c|c|c|c|c|}
\hline \multirow[t]{2}{*}{ Indicações } & \multicolumn{2}{|c|}{ Grupo 1} & \multicolumn{2}{|c|}{ Grupo 2} \\
\hline & $n$ & $\%$ & $\mathbf{n}$ & $\%$ \\
\hline Ametropia & 33 & 55,9 & 8 & 18,6 \\
\hline Anisometropia & 11 & 18,6 & 10 & 23,3 \\
\hline Leucoma & 0 & 0 & 8 & 18,6 \\
\hline Esotropia & 10 & 16,9 & 0 & 0 \\
\hline Ceratocone & 1 & 1,7 & 6 & 13,9 \\
\hline Phthisis & 0 & 0 & 7 & 16,3 \\
\hline Afacia traumática & 0 & 0 & 5 & 11,6 \\
\hline Catarata congênita & 3 & 5,2 & 2 & 4,6 \\
\hline $\begin{array}{l}\text { Anisometropia + } \\
\text { midríase paralítica }\end{array}$ & 0 & 0 & 1 & 2,3 \\
\hline Tampão & 1 & 1,7 & 0 & 0 \\
\hline $\mathrm{p}<0,0001$ & & & & \\
\hline
\end{tabular}

\begin{tabular}{|c|c|c|c|c|}
\hline \multirow{2}{*}{$\begin{array}{l}\text { Tipo de lente } \\
\text { de contato }\end{array}$} & \multicolumn{2}{|c|}{ Grupo 1} & \multicolumn{2}{|c|}{ Grupo 2} \\
\hline & $\mathbf{n}$ & $\%$ & $\mathbf{n}$ & $\%$ \\
\hline Rígida & 4 & 6,8 & 13 & 30,2 \\
\hline Gelatinosa descartável & 18 & 30,5 & 0 & 0 \\
\hline $\begin{array}{l}\text { Gelatinosa não } \\
\text { descartável }\end{array}$ & 27 & 45,8 & 14 & 32,6 \\
\hline Tórica & 10 & 17,0 & 0 & 0 \\
\hline $\begin{array}{l}\text { Filtrante com } \\
\text { pupila preta }\end{array}$ & 1 & 1,7 & 11 & 25,6 \\
\hline $\begin{array}{l}\text { Filtrante com } \\
\text { pupila transparente }\end{array}$ & 0 & 0 & 2 & 4,7 \\
\hline$p<0,001$ & & & & \\
\hline
\end{tabular}

\begin{tabular}{|c|c|c|c|c|}
\hline \multirow[t]{2}{*}{ Complicações } & \multicolumn{2}{|c|}{ Grupo 1} & \multicolumn{2}{|c|}{ Grupo 2} \\
\hline & $\bar{n}$ & $\%$ & $\mathbf{n}$ & $\%$ \\
\hline Desconforto & 4 & 13,4 & 0 & 0 \\
\hline Perda da lente & 1 & 3,4 & 3 & 60 \\
\hline Conjuntivite alérgica & 0 & 0 & 2 & 40 \\
\hline Abrasão corneana & 2 & 6,9 & 0 & 0 \\
\hline Lente trincada & 1 & 3,4 & 0 & 0 \\
\hline Fungo & 1 & 3,4 & 0 & 0 \\
\hline Olho vermelho & 1 & 3,4 & 0 & 0 \\
\hline$p<0,044$ & & & & \\
\hline
\end{tabular}

De todas as crianças analisadas apenas 3 não compraram a lente de contato após o teste, todas do grupo 2 . Cinco crianças não permitiram a realização do teste, sendo 3 do grupo 1 e 2 do grupo 2. Não foi possível adaptar a lente em 3 crianças do grupo 2, devido a irregularidade na superficie corneana.

A distribuição das frequências da acuidade visual com lente de contato comparada aos óculos, apresenta-se na tabela 4.

No grupo 2 em 14 olhos foram observados visão nula, não havendo nenhum caso no grupo 1 . Em 13 casos no grupo 1 não foi referida a acuidade visual, já no grupo 2 , em 9 casos. 


\begin{tabular}{|c|c|c|c|}
\hline \multicolumn{4}{|c|}{$\begin{array}{l}\text { Tabela 4. Distribuição das frequências da acuidade visual } \\
\qquad \text { AV com lente de contato comparada a óculos }\end{array}$} \\
\hline & Manteve & Melhorou & Piorou \\
\hline \multicolumn{4}{|l|}{ Grupo 1} \\
\hline OD & 35 & 3 & 4 \\
\hline OE & 33 & 2 & 8 \\
\hline \multicolumn{4}{|l|}{ Grupo 2} \\
\hline OD & 7 & 2 & 6 \\
\hline OE & 6 & 3 & 5 \\
\hline
\end{tabular}

DISCUSSÃO

Nesse estudo a indicação mais frequente nos dois grupos foi ametropia, o que condiz com a literatura ${ }^{(6)}$. A indicação mais frequente no hospital privado foi ametropia, e no público anisometropia. O leucoma, a catarata traumática e a phthisis apareceram de maneira significante no grupo 2, não sendo relatado no grupo 1. Estes achados dão suporte à possibilidade de maior quantidade de úlcera de córnea ou trauma ocular nos pacientes atendidos em serviços públicos. A indicação do uso de lente de contato por esotropia foi observada apenas no grupo 1, talvez devido ao mais baixo custo dos óculos bifocais comparados às lentes de contato prescritas no grupo 1 .

A lente de contato mais frequentemente indicada nos dois grupos foi a gelatinosa de uso permanente (não descartável), fato não observado na literatura em que a lente rígida se constitui a mais indicada ${ }^{(1,6)}$. Esta indicação deve-se ao fato das crianças aceitarem melhor as lentes gelatinosas ${ }^{(1)}$. A lente gelatinosa de uso descartável não foi prescrita no serviço público, devido ao seu mais alto custo comparado às lentes de uso permanente. Trabalhos mostram a boa aceitação das lentes descártaveis de uso prolongado, como as silicone hidrogel, em crianças com fins terapêuticos ${ }^{(7)}$. Deve-se levar em consideração o uso dessas lentes nas ametropias em crianças devido ao seu alto Dk, o que elimina a hipóxia induzida pela lente na maioria dos usuários ${ }^{(8)}$, e devido ao seu uso contínuo. As lentes silicone hidrogel não foram usadas nesse estudo, pois como esse estudo foi retrospectivo, essas lentes ainda não estavam difundidas no Brasil.

A acuidade visual nos dois grupos, comparando-se o uso de lente de contato com óculos, manteve-se, na maioria dos casos, similar à encontrada na literatura. Fato de grande importância, pois comprova a boa aceitação das crianças à lente, sendo especialmente bem indicada para crianças com altos vícios de refração que desejam praticar esportes e o uso dos óculos não permite, além da lente de contato proporcionar melhor qualidade de visão ${ }^{(9)}$.

O número de complicações observadas foi pequeno, especialmente no que se refere à perda da lente, possivelmente devido a falta de informação do paciente ou deficiência no seguimento. O maior número de complicações nos pacientes do serviço privado, provavelmente reflete a maior facilidade de retorno destes pacientes ao médico sempre que surgem as queixas.

\section{CONCLUSÃO}

As indicações do uso de lente de contato em crianças de serviços público e privado foram diferentes, principalmente devido a ausência de leucoma, phthisis e afacia traumática como indicações no serviço privado. $\mathrm{O}$ tipo de lente de contato mais prescrita nos dois serviços foi a gelatinosa não descártavel, não havendo lente descartável no serviço público. Ocorreu o maior número de complicações nos pacientes do serviço privado. A acuidade visual manteve-se na maioria dos casos com lente de contato.

\section{ABSTRACT}

Purpose: To analyze the indications, type and complications of contact lens use and visual acuity in children, in ophthalmological, public and private, services. Methods: The information from the medical records of 59 contact lens users at a private service (Hospital de Olhos de Pernambuco - Recife - PEBrazil - group 1), and 43 at public service (Fundação Altino Ventura - Recife - PE - Brazil - group 2), was analyzed. The collected data included: demographic information; age at first examination; indication of lens use; contact lens type; complications and visual acuity. Results: The most common indications of contact lenses in group 1 were: ametropia (55.9\%), anisometropia (18.6\%) and esotropia (16.9\%). In this group leukoma and phthisis were not present. In group 2 the most common indications were: anisometropia (23.2\%), ametropia $(18.6 \%)$, leukoma (18.6\%) and phthisis (16.3\%). Esotropia was not found in group 2 . The most prescribed contact lens was soft and of permanent use in group $1(45.8 \%)$ and in group $2(32.6 \%)$. The most frequent complication in group 1 was discomfort $(33.3 \%)$ and in group 2 was the loss of the lens (60\%). Conclusions: The most frequent indication in private services was ametropia and anisometropia in the public ones. The type of lens mostly prescribed in both groups was soft and of permanent use. The most frequent complication in group 1 was discomfort and in group 2 loss of the lens. The visual acuity was the same in the majority of the patients.

Keywords: Contact lens/adverse effects; Visual acuity; Child; Hospitals, public; Private service; Hospitals, private; Comparative analysis

\section{REFERÊCIAS}

1. Moreira SMB, Moreira H, Moreira LB. Adaptação de lentes de contato em crianças. In: Moreira SMB, Moreira H, Moreira LB. Lentes de contato. $2^{\underline{a}}$ ed. Rio de Janeiro: Cultura Médica; 1998. p.235-7.

2. Moore B. Pediatric contact lenses: case reports. J Am Optom Assoc. 1998; 69(2):88-114. Erratum in: J Am Optom Assoc 1998;69(3):142. Comment in: J Am Optom Assoc. 1998;69(7):452.

3. Soares EF. Lentes de contato em crianças. An Oftalmol. 1985;4(1):79-81.

4. Burger DS, London R. Soft opaque contact lenses in binocular vision problems. J Am Optom Assoc. 1993;64(3):176-80.

5. Jurkus JM. Contact lenses for children. Optom Clin. 1996;5(2):91-104. 
6. Coral-Ghanem C. Lentes de contato em pediatria. In: Coral-Ghanem C, KaraJosé N. Lentes de contato na clínica oftalmológica $2^{\underline{a}}$ ed. Rio de Janeiro: Cultura Médica; 1998. p.85-91.

7. Ozkurt Y, Rodop O, Oral Y, Cömez A, Kandemir B, Dogan OK. Therapeutic applications of lotrafilcon a silicone hydrogel soft contact lenses. Eye Contact Lens. 2005;31(6):268-9.
8. Stapleton F, Stretton S, Papas E, Skotnitsky C, Sweeney DF. Silicone hydrogel contact lenses and the ocular surface. Ocul Surf. 2006;4(1):24-43. Review.

9. Stenson SM. Pediatric contact lens fitting. In: Kastl PR, editor. Contact lenses. The CLAO guide to basic science and clinical practice. Iowa: Kendall/Hunt Publishing Company; 1995. p.179-95. 